\title{
Nuclear Level Density Calculation of Astrophysical Reactions
}

\author{
Ahmed A. Selman ${ }^{1}$, Hamsa S. Jasim ${ }^{2}$ \\ ${ }^{I}$ Department of Astronomy and Space, College of Science, Baghdad University, Baghdad, Iraq \\ ${ }^{2}$ Department of Astronomy and Space, College of Science, Baghdad University, Baghdad, Iraq
}

\begin{abstract}
Nuclear state density was calculated for the nuclear reaction inside the core of three different mainsequence stars namely, Sun, Sirius and Vega. The selected nuclei for present calculation were $\mathrm{H}^{4}, \mathrm{C}^{12}, \mathrm{~N}^{14}$, and $\mathrm{O}^{16}$; and the state density of $\mathrm{Fe}^{54}$ nucleus was also included for comparison. The state density was calculated based on the exciton theory of nuclear reaction from Ericson, Williams and pairing formulae. The results confirmed that any change in the configuration will result different values of state density and the results showed that the dependence on energy is not linear, and higher densities were found at higher energies, but the change was less.
\end{abstract}

\section{Introduction}

The physics of nuclear structure and nuclear reactions is entering a new field, due to the development of radioactive nuclear beams, which enable us to study a variety of nuclei far from stability. A nuclear reaction caused by an incident particle result in sharing of the incident particles energy with all nucleons within the nucleus to reach the final stage is called "Thermal Equilibrium State". The nucleus at this stage is called "The Compound Nucleus". In between direct and $\mathrm{CN}$ emissions, there is an intermediate and continuous emission of particles which is called the

"PE.". Griffin [2] in 1966 proposed the exciton model, which is a semi-classical approach to explain $\mathrm{PE}$, Being a simplified model. An important parameter in PE theory is the state density. The importance of the state and level densities rise from its ability to describe population of nuclear excited states. The aim is to try applying the exciton model on stellar reactions. Studies of the solar structure have shown that the main energy it radiates comes from a series of nuclear reactions called the $p-p$ chain reaction and $\mathrm{CNO}$ cycle [1-7].

\section{The Exciton Model}

Griffin [2] assumed the precompound nucleus will exist with formed "Excitons". the main reasons behind the popularity of Griffin's exciton model are [3]:

1. Exciton creation means creating the pair (particle +hole), the particle being excited above $F$.

2. Each exciton state may decay with a time rate that depends on the exciton number, $n$, and excitation energy, $E$. In nuclear reactions, this must be the case because the decay rate must depend on number of excited particles (population of excited particle states) and excitation energy.

3. When many numbers of excitons are created, the strength of the exciton creation process becomes less significant. This means that as the excitation states developed and grow in number, exciton creation becomes less effective due to the reduction of the excitation energy which lowers the chances of exciton creation.

4. There is a maximum exciton number $n_{\max }$ which represents the final possible exciton state. In nuclear reactions, also a final excitation state must exist. At $n_{\max }$, no further exciton creation is possible. In terms of nuclear reactions, it is said that the nucleus reached thermal equilibrium and the $\mathrm{CN}$ is formed.

5. Thus, choosing exciton idea to represent nuclear reactions is quite suitable.

\section{Nuclear Reactions in Main Sequence Stars}

The solar structure has shown a series of nuclear reactions called the $p$ - $p$ chain and $\mathrm{CNO}$ chain reactions [10-12]. The p-p reaction comes from a series of the following individual reactions or steps:

$$
\begin{gathered}
{ }_{1} \mathrm{H}+{ }_{1} \mathrm{H} \rightarrow{ }_{2} \mathrm{H}+\mathrm{e}^{+} \\
{ }_{2} \mathrm{H}+{ }_{1} \mathrm{H} \rightarrow{ }_{3} \mathrm{He}+\text { photon } \\
{ }_{3} \mathrm{He}+{ }_{3} \mathrm{He} \rightarrow{ }_{4} \mathrm{He}+{ }_{1} \mathrm{H}+{ }_{1} \mathrm{H}
\end{gathered}
$$

The first and third reactions could take $10^{6}$ years to start, while the second step occurs within $10^{-3} \mathrm{sec}$. Each step of these produces a certain amount of energy from mass to energy conversion $E=m_{0} c^{2}$, because the helium nucleus weighs less than the sum of the four protons. Energy emerges in three forms: neutrinos that flee the Sun's core at the speed of light, protons that participate in a new round of fusion, and photons that percolate 
through the Sun (still at the speed of light) until they leave the Sun's surface as visible light. CNO is a fusion process that combines protons to form helium nuclei through intermediary nuclei of carbon, nitrogen, and oxygen. This process requires a temperature of about 15 million $\mathrm{K}$, which is higher than the core temperature of the Sun, but is less efficient than the Sun's p-p chain reaction fusion reaction. The reactions are as follows:

$$
\begin{gathered}
{ }_{12} \mathrm{C}+{ }_{1} \mathrm{H} \rightarrow{ }_{13} \mathrm{~N}+\text { photon } \\
{ }_{13} \mathrm{~N} \rightarrow{ }_{13} \mathrm{C}+\mathrm{e}^{+}+\text {neutrino } \\
{ }_{13} \mathrm{C}+{ }_{1} \mathrm{H} \rightarrow{ }_{14} \mathrm{~N}+\text { photon } \\
{ }_{14} \mathrm{~N}+{ }_{1} \mathrm{H} \rightarrow{ }_{15} \mathrm{O}+\text { photon } \\
{ }_{15} \mathrm{O} \rightarrow{ }_{15} \mathrm{~N}+\mathrm{e}^{+}+\text {neutrino } \\
{ }_{15} \mathrm{~N}+{ }_{1} \mathrm{H} \rightarrow{ }_{12} \mathrm{C}+{ }_{4} \mathrm{He}
\end{gathered}
$$

The process, therefore, includes consuming four hydrogen atoms. The carbon cycle requires high temperature to operate, which can only found in the core of massive stars [1].

\section{The State Density}

The state density is an important quantity where the main calculations are based on. The EquidistantSpacing Model (ESM) approximation of the nuclear state density is described by the single particle state density $g$. In ESM, the energy difference between any two successive energy levels is $\Delta E_{\mathrm{n}}=1 / g=$ constant [4]. The exciton number, $n$, is defined as $n=p+h$,

when it is assumed that protons and neutrons are indistinguishable particles. For a two-component Fermi gas system, we have $n=$ $n_{\pi}+p_{\pi}+h_{\pi}+p_{v}+h_{v}$. The state density for one-component Fermi gas system will be denoted by $\omega_{1}(n, E)$ and for two-component by $\omega_{2}(n, E)$. The state density calculation includes the following treatments:

1- Un-corrected (Ericson) formula,

2- Pauli blocking (Williams Formula),

3- Back-shifted levels and modified Pauli terms,

4- Pairing correction.

\subsection{Ericson's Formula}

The simplest formula used to describe $\omega(n, E)$ is to exclude all possible corrections during the calculations, and it is called Ericson Formula - see [3]. This formula shows that state density is given for onecomponent system by,

$\omega_{1}(n, E)=\frac{g^{n} E^{n-1}}{p ! h !(n-1) !}$

Where $g$ is the single particle level density [5] $\mathrm{g}=\frac{3 A}{2 F}\left(\mathrm{MeV}^{-1}\right)$ or $\quad g \cong \frac{A}{13}$ to $\frac{A}{15}$, which is used in most practical calculations. For two-component system, the state density is given as,

$$
\omega_{2}(n, E)=\frac{g^{n} E^{n-1}}{p_{\pi} ! h_{\pi} ! p_{v} ! h_{v} !(n-1) !}
$$

which is written assuming that $g$ is the same for protons and neutrons. Since for neutrons $g_{v}$ it is different from that of protons $g_{\pi}$, therefore eq.(2) becomes [5],

where, $\quad g_{\pi} \cong \frac{Z}{A} g, g_{v} \cong \frac{(A-Z)}{A} g=\frac{N}{A} g$

\subsection{Williams' Formula}

The effect of Pauli correction appears as if the magnitude of the excitation energy was lowered. This means that several states are blocked, thus their contribution in the amount of the excitation energy will decrease. A net effect will be as if $E$ becomes $\left[E-A_{\mathrm{p}, \mathrm{h}}(p, h, E)\right]$, where $A_{\mathrm{p}, \mathrm{h}}(p, h, E)$ is Pauli correction term [6], that is to use the formula,

$$
\omega_{1}(E, n)=\frac{g^{n}\left(E-A_{p, h}\right)^{n-1}}{p ! h !(n-1) !} \Theta\left(E-\alpha_{p, h}\right)
$$


Where,

$\omega_{1}(\mathrm{E}, \mathrm{n})=$ State density of the system defined by the state of $\mathrm{P}, \mathrm{h}$ and $\mathrm{E}$, for one-component .

$\mathrm{g}=$ Single- particle level at Fermi energy.

$E=$ Excitation energy.

$A_{p, h}=$ Pauli blocking energy for one- component system .

$\alpha_{\mathrm{p}, \mathrm{h}}=$ Correction term for state density formula.

$P=$ Particle exciton number for one-component.

$h=$ Hole exciton number for one-component.

$n=$ Exciton number. $n=p+h$.

where $\Theta\left(E-\alpha_{\mathrm{p}, \mathrm{h}}\right)$ is the Heaviside step function defined as,

$$
\Theta\left(E-\alpha_{p, h}\right)= \begin{cases}0 & E-\alpha_{p, h} \leq 0 \\ 1 & E-\alpha_{p, h}>0\end{cases}
$$

And the correction term $\alpha_{\mathrm{p}, \mathrm{h}}$ is given as [6],

$$
\alpha_{p, h}=\frac{p(p+1)+h(h-1)}{2 g}
$$

For one-component. For two-component it is given as,

$$
\begin{aligned}
& \omega_{2}(n, E)=\frac{g_{\pi}^{n_{\pi}} g_{v}^{n_{v}}\left(E-A_{p_{\pi}, h_{\pi}, p_{v}, h_{v}}\right)^{n-1}}{p_{\pi} ! h_{\pi} ! p_{v} ! h_{v} !(n-1) !} \Theta\left(E-\alpha_{p_{\pi}, h_{\pi}, p_{v}, h_{v}}\right) \\
& \alpha_{p_{\pi}, h_{\pi}, p_{v}, h_{v}}=\frac{p_{\pi}\left(p_{\pi}+1\right)+h_{\pi}\left(h_{\pi}-1\right)}{2 g_{\pi}}+\frac{p_{v}\left(p_{v}+1\right)+h_{v}\left(h_{v}-1\right)}{2 g_{v}}
\end{aligned}
$$

\section{Where,}

$\omega_{2}(\mathrm{E}, \mathrm{n})=$ State density of the system defined by the state of $\mathrm{P}, \mathrm{h}$ and $\mathrm{E}$, for two-component.

$\mathrm{g}_{\pi}\left(\right.$ or $\left.\mathrm{g}_{\mathrm{v}}\right)=$ Single-Particle level density for proton (or neutron).

$h_{\pi}\left(\right.$ or $\left.h_{v}\right)=$ Hole exciton number for proton (or neutron) particle for two-component.

$P_{\pi}\left(\right.$ or $\left.P_{v}\right)=$ Particle exciton number for protons (or neutrons) for two-component.

$A_{p \pi, h \pi_{i} p v_{s} h v}=$ Pauli blocking energy for two-component system.

\subsection{Pairing and Modified Williams' Formula with Back-Shift Fermi Gas:}

Correction due to pairing will also cause a reduction in the excitation energy $E$ by the amount of energy, $\Delta$, that is generated from pairing effect. So, the "Effective Excitation Energy", $E_{\text {eff }}$, of the system will be, $E_{\text {eff }}=E-\Delta$, then the general form, $E_{\text {eff }}=E-f(\Delta)$ Where, $f(\Delta)$ is a correction function of energy that depends in one way or another on pairing, which is given for energy back-shift. Using the ESM approximation, then $\omega_{1}(n, E)$ of a system will be given as [3]

$$
\omega_{1}(n, E, P)=\frac{g^{n}\left(E-P(\Delta)-B_{p, h}\right)^{n-1}}{p ! h !(n-1) !} \Theta\left(E-P(\Delta)-B_{p, h}\right)
$$

Where, $P(\Delta)=g \frac{\left(\Delta_{0}^{2}-\Delta^{2}\right)}{4}, B_{p, h}$ the modified Pauli energy and the energy gap $\Delta$ is given as [3], And $E_{p h a s e}$ is the pairing energy for phase transition [3] defined as following,

$$
\omega_{1}\left(n, E, A_{K}\right)=\frac{g^{n}\left(E-A_{K}(p, h)\right)^{n-1}}{p ! h !(n-1) !}
$$

The simplest Back-Shift Femi Gas model (BSFG) equation for one-component is more appropriate to include the Heaviside step function so that [3], 
$\omega_{1}(n, E, S)=\frac{g^{n}(E-P(\Delta)-B-S)^{n-1}}{p ! h !(n-1) !} \Theta(E-P(\Delta)-B-S)$

where $S$ is the Back-Shift energy.

\section{Results and Discussions}

Nuclear state density is calculated for the nuclear reaction inside the core of three different main-sequence stars, namely: Sun, Sirius and Vega. In Table (1) the general specifications of these stars are listed.

Table (1).General specifications of selected stars

\begin{tabular}{|l|l|l|l|l|l|l|l|l|l|}
\hline Star & $\begin{array}{l}\text { Mass } \\
\mathrm{M}_{\mathrm{s}}^{*}\end{array}$ & $\begin{array}{l}\text { Age } \\
\mathrm{x} 10^{9} \mathrm{yr}\end{array}$ & $\begin{array}{l}\text { Distance } \\
(\mathrm{average})\end{array}$ & $\begin{array}{l}\text { Diameter** } \\
\mathrm{R}_{\mathrm{s}}\end{array}$ & Abs. Mag. & Temp. K & $\mathrm{L}$ & Spectrum & Color Index \\
\hline Sun & 1 & 4.57 & $1 \mathrm{AU}$ & 1 & -26.8 & 5778 & $\begin{array}{l}3.75^{*} 10^{62} \\
\text { Lumn }\end{array}$ & $\mathrm{G} 2 \mathrm{~V}$ & Yellow \\
\hline Vega & 2.1 & $\sim 1$ & $25.0 \mathrm{ly}$ & 2.7 & +0.59 visual & 9790 & 49 suns visual & A0Va & 0.00 White \\
\hline Sirius & 2.1 & 0.240 & $8.58 \mathrm{ly}$ & 1.93 & +1.46 visual & 9400 & 22.1 suns visual & A1Vm & 0.02 White + \\
\hline
\end{tabular}

* $1 \mathrm{M}_{\mathrm{s}}=1.999 \times 10^{30} \mathrm{~g}$

$* * 1 \mathrm{R}_{\mathrm{s}}=1.391 \times 10^{6} \mathrm{~km}$

It can be seen from Table (1) that both their ages are less than the Sun and have more surface temperature..etc. In this research the attention will be focused on state density calculations for reactions using the necessary elements in the selected stars, namely, the nuclei of $\mathrm{He}^{4}, \mathrm{C}^{12}, \mathrm{~N}^{14}$, and $\mathrm{O}^{16}$. For comparison purpose, the state density of $\mathrm{Fe}^{54}$ nucleus will also be included. Only few state density corrections will be used. In all calculations made below, the ESM approximation is assumed.

Table (2). Input values of state density calculations for ${ }_{2}^{4} \mathrm{He} e_{2},{ }_{6}^{12} \mathrm{C}_{6}{ }_{7}^{14} \mathrm{~N}_{7},{ }_{8}^{16} \mathrm{O}_{8}$ and ${ }_{26}^{54} \mathrm{Fe}_{28}$ nuclei.

\begin{tabular}{|l|l|}
\hline$E_{\max }$ & $100 \mathrm{MeV}$ \\
\hline$n_{\max }$ & 4 to 6 \\
\hline one-component $(\mathrm{p}, \mathrm{h})$ & $(3,2)$ \\
\hline two-component $\left(\mathrm{p}_{\pi}, \mathrm{h}_{\pi}, \mathrm{p}_{\mathrm{v}}, \mathrm{h}_{\mathrm{v}}\right)$ & $(3,2,0,0)$ or $(3,1,0,1)$ \\
\hline Single particle density, $g$ & $A / 13\left(\mathrm{MeV}^{-1}\right)$ \\
\hline Back-shift energy, $S$ & $5 \mathrm{MeV}$ \\
\hline Pairing correction, $U_{\mathrm{p}}$ & $3.5 \mathrm{MeV}$ \\
\hline & Proton: $8 \mathrm{MeV}$ \\
Nucleon's binding energy, $B$ & Neuron: $10 \mathrm{MeV}$ \\
\hline Fermi Energy, $F$ & Proton: $38 \mathrm{MeV}$ \\
& Neutron: $40 \mathrm{MeV}$ \\
\hline Potential depth, $V$ & $38 \mathrm{MeV}$ \\
\hline
\end{tabular}

\subsection{State density calculation with no corrections, Ericson's formula}

Results of one-component and two-component nuclear state density calculated using Ericson's formula (with no corrections) are shown in Fig.(1) the one-component results are shown for $\mathrm{A}=4,12,14,16$ and 54; corresponding to nuclei $\mathrm{He}^{4}, \mathrm{C}^{12}, \mathrm{~N}^{14}, \mathrm{O}^{16}$ and $\mathrm{Fe}^{54}$ respectively. The configuration was fixed at $(\mathrm{p}, \mathrm{h})=(3,2)$. The results clearly show that as $\mathrm{A}$ increases, the value of $\mathrm{g}$ will also increase and the state density also increase. The results for two-component with configuration $\left(p_{\pi}, h_{\pi}, p_{v}, h_{v}\right)=(3,2,0,0)$ are shown in Fig.(2).

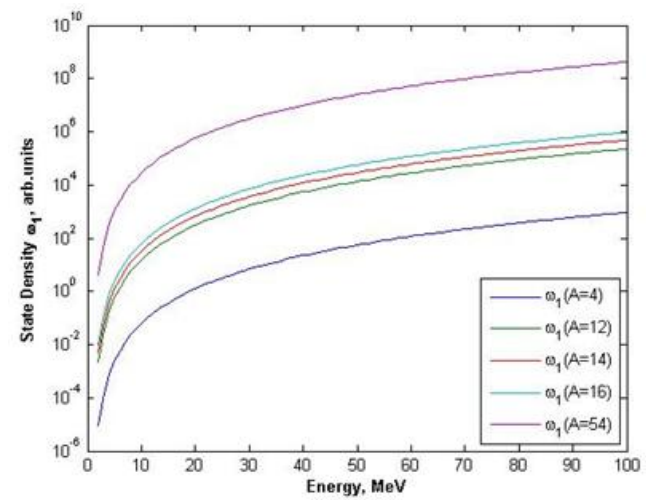

Fig.(1). The results of the state density $\omega_{1}$ as a function of excitation energy, for one-component using Ericson's formula. The configuration was $\mathrm{p}, \mathrm{h}=(3,2)$ and $\mathrm{A}=(4,12,14,16,54)$ 


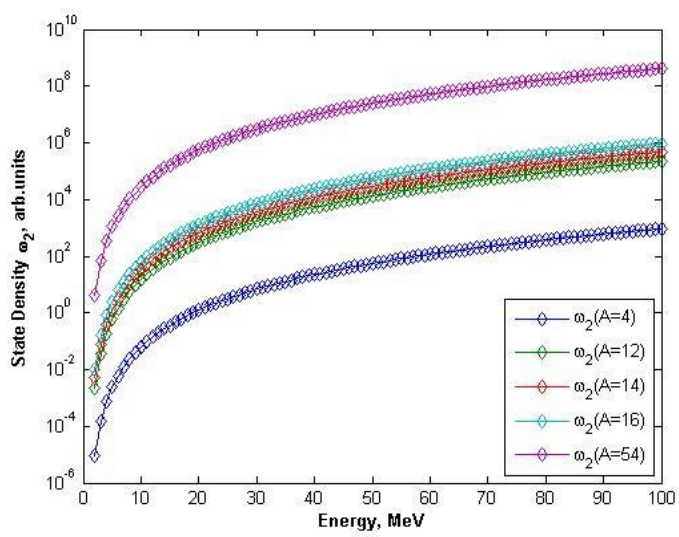

Fig.(2). The results of the state density $\omega_{2}$ as a function of excitation energy, for two-component using Ericson's formula

\subsection{State density calculations with Pauli correction, Williams' Formula}

The results in Fig.(3) are of state density for one-component that did not use Heaviside formula of eq.(5). Therefore it is seen that there is a sudden drop in the state density at lower energies. At energy $\mathrm{E} \sim 10$ $\mathrm{MeV}$ the line dropped for $\mathrm{A}=4$, and as $\mathrm{A}$ increase the drop point is shifted toward lower energies reaching $~ 1$ $\mathrm{MeV}$ at $\mathrm{A}=54$. This behavior was not seen in other work such as [3]. The same calculation was made but for two-component in Fig.(4). An important remark here is that the behavior dropped at higher energies than in onecomponent results. For $\mathrm{A}=4$, the line dropped at $\mathrm{E} \sim 18 \mathrm{MeV}$ and at $\mathrm{A}=54$ at $\mathrm{E} \sim 5 \mathrm{MeV}$. The state density is calculated based on eq. (4) and (6), with the Heaviside function, and the results are shown in Fig.(5) for one component, and in Fig.(6) for two-component respectively. The state density is was smoother with increasing energy than before and there was no sudden drop in the results. The Heaviside function actually cut the results lower than Pauli blocking $A_{p}$ energy and forced the state density to start with values higher than this.

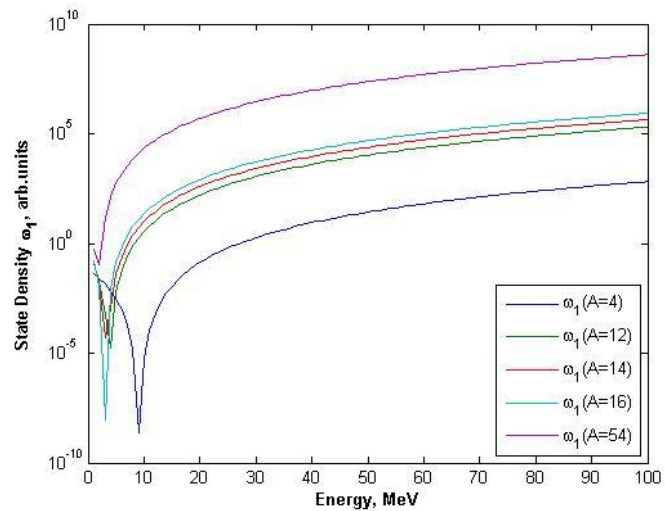

Fig.(3). The results of one-component state density as a function of Energy based on Williams' formula without Heaviside function. The configuration is $(3,2)$.

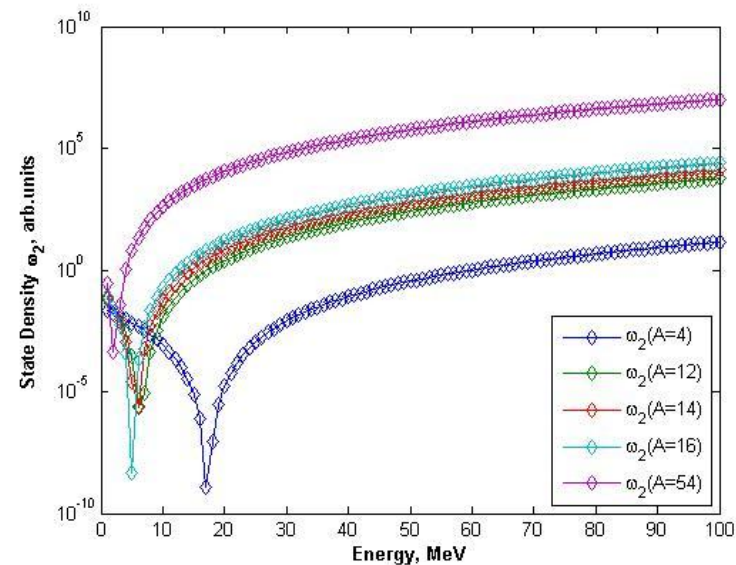

Fig.(4). The same as Fig.(3) for two-component system based on Williams' formula without Heaviside function. 


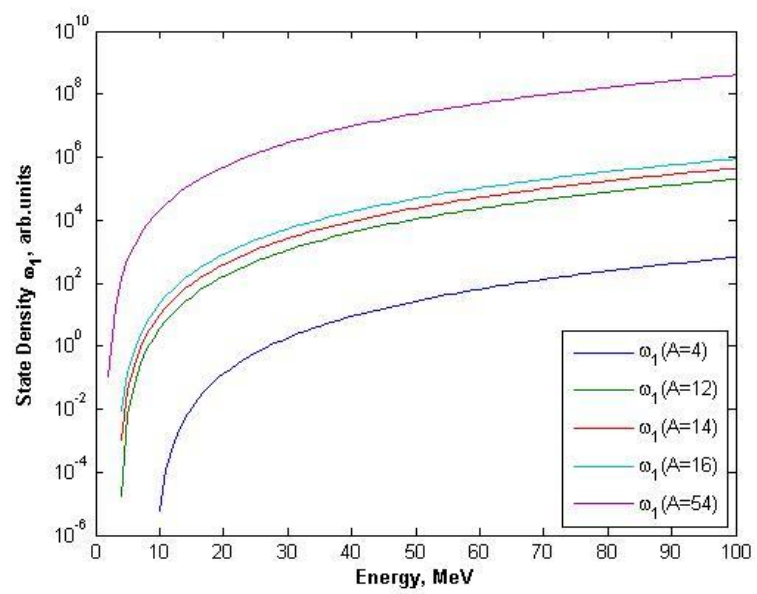

Fig.(5). The results of one-component state density as a function of Energy based on Williams' formula with Heaviside function. The configuration is $(3,2)$.

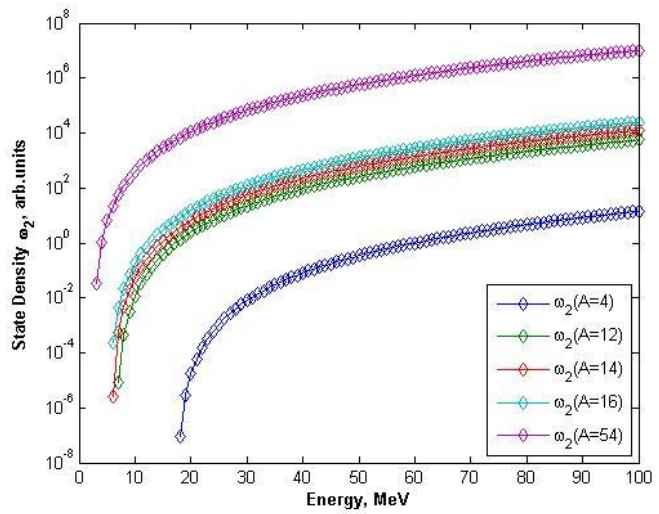

Fig.(6). The same as Fig.(5) for two-component based on Williams' formula with Heaviside function. The configuration is $(3,2,0,0)$.

Furthermore, a comparison between Ericson's and Williams' formulae is made by means of percentage difference. The formula used is:

Ratio $=\frac{\left(\omega_{\text {Ericson }}-\omega_{\text {Williams }}\right)}{\omega_{\text {Ericson }}}$

for both one-component and two-component. This calculation was made in order to study the effect of adding Pauli energy and the Heaviside function on both types of calculations. The results are shown in Fig.(7) for onecomponent, and in Fig.(8) for two-component; with similar input as before.

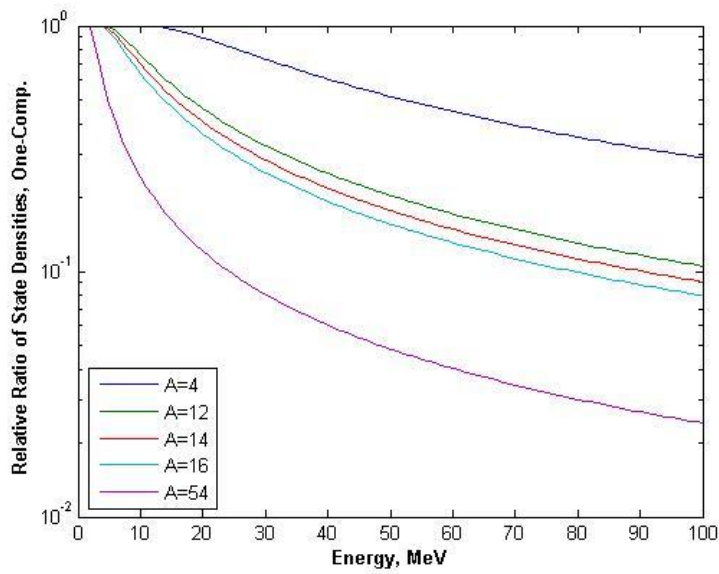

Fig.(7). A percentage comparison between one-component state density for Ericson and Williams formulae. All input values were the same. 


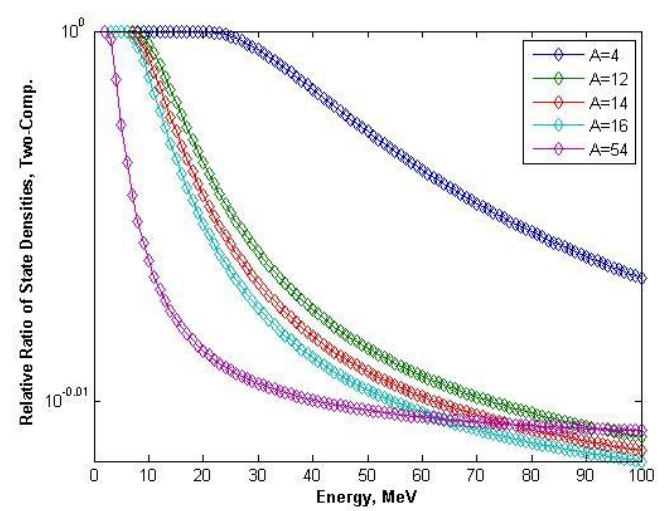

Fig.(8). A percentage comparison between two-component state density for Ericson and Williams formulae. All input values were the same.

\subsection{Paring correction:}

The calculated state density as a function of E using the pairing formula eq.(9) for the one-component configuration $(3,2)$ is shown in Fig.(9), for the selected range of mass numbers. Configuration was $(3,2)$ in both cases. This effect appears at low energy range and decreases at higher energies, because the pairing effect represents the resistance to the excitation process. It can be seen that as the mass number $A$ increases, the pairing effect decreases. The maximum effect was at $A=4$, the helium nucleus. Also from Fig.(9) there is a cut-off in state density for all cases, specially for helium nucleus and as $A$ increase this cut-off decreases. The pairing effect on the state density calculations which are based on eq.(10) is shown in Fig.(10) with back-shift and no other modification. From comparing Fig.(9) with Fig.(10) it can be seen that at $A=4$, there was a large change in the state density, where it started from E $60 \mathrm{MeV}$. This is because of the back-shift of energy which reduces the state density at lower energies. Such behavior may prevent the helium nucleus from going through fusion reaction due it's higher binding energy.

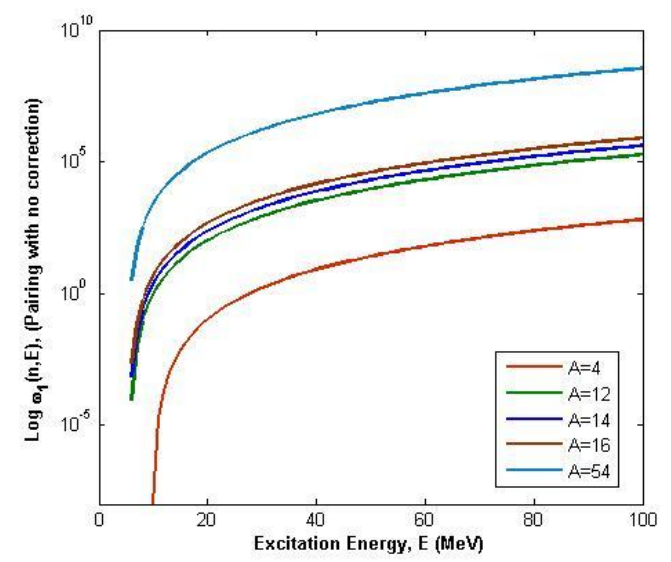

Fig.(9). The calculated state density as a function of $\mathrm{E}$ for one-component system with paring correction. No other modification was used - eq.(9).

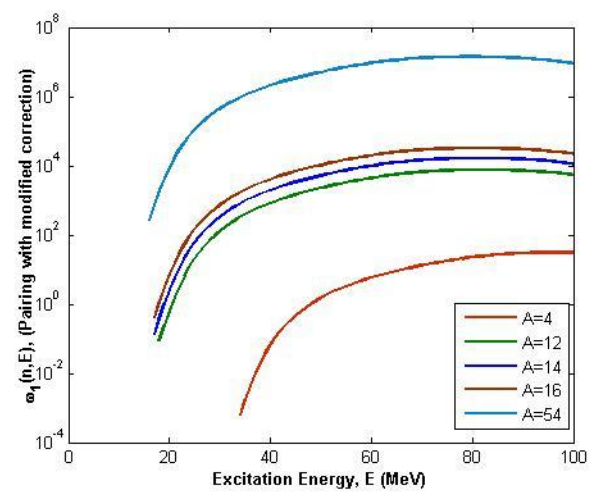

Fig.(10). The calculated state density as a function of $\mathrm{E}$ for one-component system with paring correction and no back-shift energy. Modified correction- eq.(10). 
In Fig.(11) the pairing effect with BSFG is shown from eq.(11). In this case the effect of back shift energy $S$ will further reduce the energy share by each exciton state, therefore the overall values will decrease more than in the previous cases.

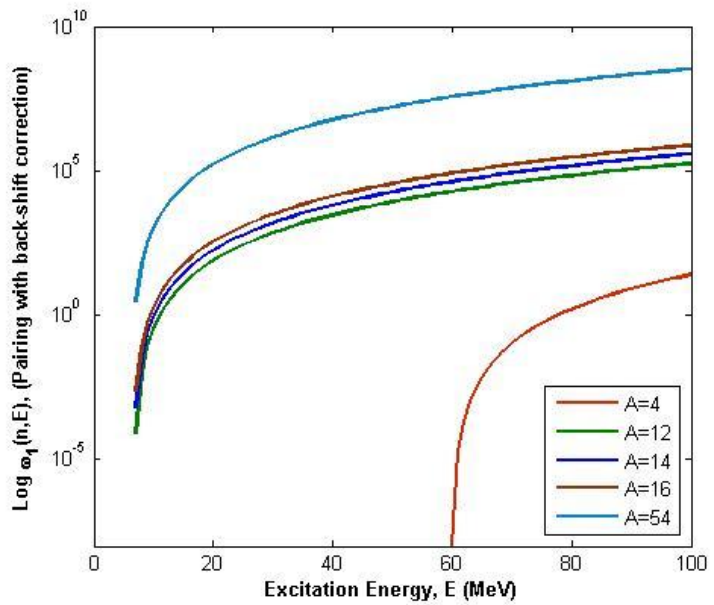

Fig.(11). The calculated state density as a function of $\mathrm{E}$ for one-component system with paring correction and back-shift energy- eq.(11).

The effects of $S$ and, $B$ on state density for one-component show in Figs.(9) to (11). The state density results change with the value of $B$ where as it increases the state density increases with fixing everything else during the calculations. For large values of binding energy $B$, the state density results are almost identical except at the end, where a slight reduction occurred. However at lower $B$ values, there will be higher changes. This indicates that state density calculations at $B$ is more close to realistic situation and that is very sensitive to this value. Also at $B=8 \mathrm{MeV}$, the result will be very different from the values above $10 \mathrm{MeV}$, and $B=2 \mathrm{MeV}$ as well. Furthermore, in Fig.(12) the calculations were made for the configuration $p=h=2$. It is preferred to use $p=h$ for cases of closed-shell nuclei as mentioned in [3] because in the case of closed-shell nuclei there is a higher chance for both particle and hole similarities

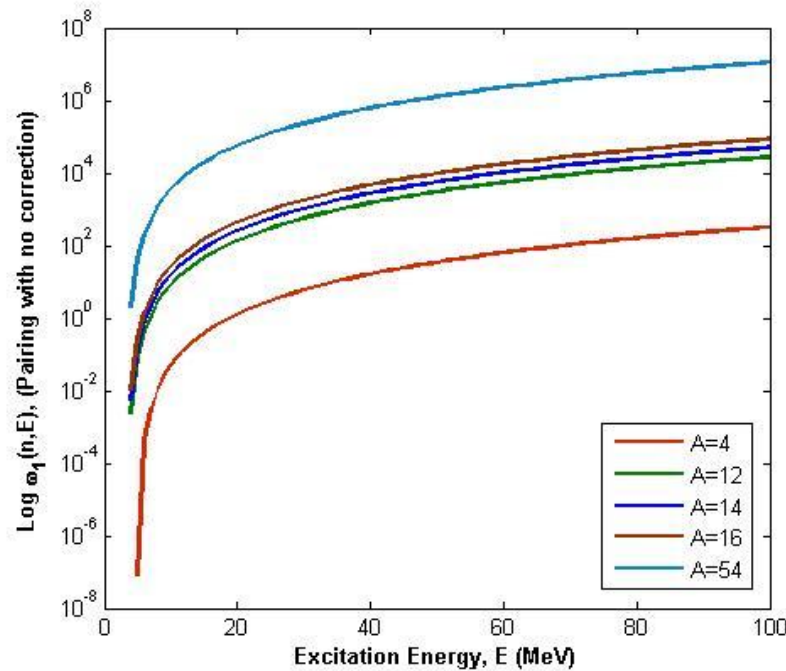

Fig.(12). The calculated state density as a function of $\mathrm{E}$ for one component system with paring correction. No other modification was used - eq.(9).Configuration was $p=h=2$.

A calculation is made of state density based on paring with William's formula for same exciton number and for the same nucleus $\mathrm{He}^{4}$ in Fig.(13). This figure is made in order to show the total effect of pairing on Williams' formula, based on eq.(11). The configuration was assumed to be $p=h$ with values from 1 and 2 . Also the summation of the state densities is shown. The summation of state densities plays important role in calculating the reaction cross-section [3]. 


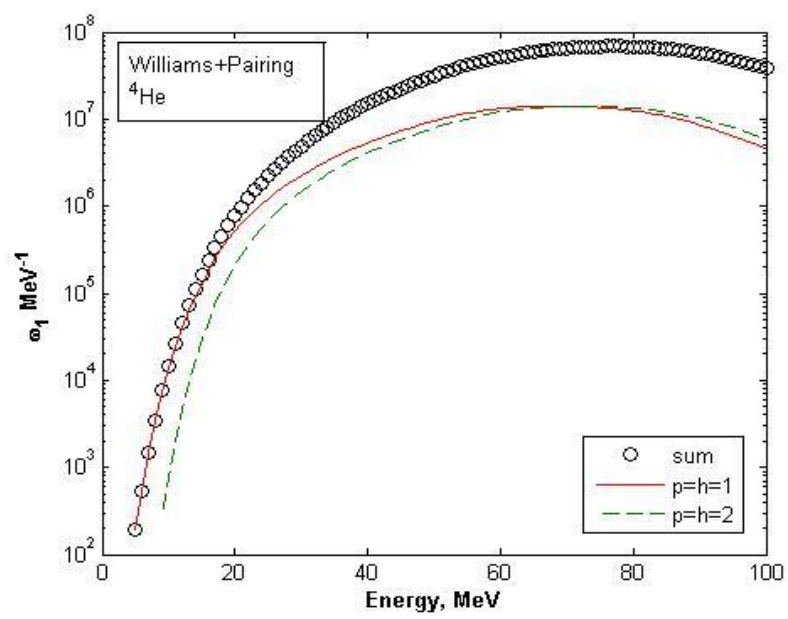

Fig.(13). The calculated state density as a function of Energy for one-component system with paring correction and back-shift energy. The configuration $p=h$ is used for cases 1 and 2 . The summation is also given.

The set of figures from Fig.(9) to Fig.(13) all used various physical parameters. These parameters are calculated within the program codes for each case.

\subsection{State Density with 3-D Plots}

In order to represent the behavior of state density with different exciton numbers $n$, for a range of energy $E$, three-dimensional plots were made for Ericson and Williams' formulae below. In the case of Williams' formula the two-component results are less than the one-component results because the Pauli correction term becomes less, as shown in Fig.(14).

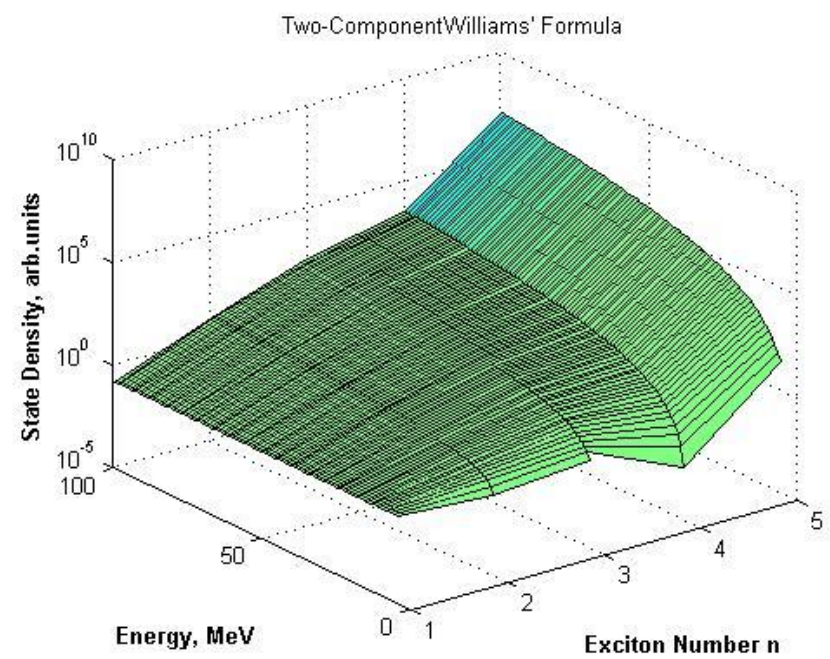

Fig.(14). Three-dimensional plot of the state density based on two-component Williams' formula. A fixed value of $\mathrm{A}=4$ was used in this figure.

The following conclusions can be made:

\section{Conclusions}

1. From Ericson formula study of the state density, it was confirmed that any change in the configuration will result different values of state density and, the results showed that the dependence on energy is not linear. Also in the state density with Ericson formula, the values of higher densities were found at higher energies, but the change was less.

2. Also from Williams' an important remark was seen from that the configurations $(3,2,0,0)$ and $(3,2)$, although were the same with proton particles and holes, but they resulted in different behavior at the same energies. This is because of Pauli correction which blocks some of the energy for the exciton.

3. Comparison between Ericson and Williams formulae shoes that 
a) State densities for one-component are generally higher than those of two-components for the same configuration and other input values,

b) Ericson's formula gave in higher state density values than Williams' formula,

c) As the energy increases the differences increase between one-component and two-component, and the differences also increase between Ericson and Williams formulae,

d) As the mass number A increases, the percentage ratio decreases in favor of Ericson formula. There was a notable behavior of two-component state density for Fe nucleus where its values went higher than $\mathrm{A}=12$ 16 at energies above $\sim 90 \mathrm{MeV}$. This indicates that this nucleus has more reaction capability at higher energies.

4- The overall comparison with exciton number and energy showed that most probable reaction will be at higher energies and higher exciton numbers.

5- Pairing results also showed that selecting the case of $p=h=2$ the state density was with lower values than in the case $p=3, h=2$. The total exciton number also decreases which leads to less state density. This was because of the distribution of energy on less components which gives larger share for each particle or hole, thus the state density will increase in the case of different $p$ and $h$.

6- A general remark that was reached is that: less state density means less reaction probability.

\section{References}

[1]. $\quad$ Burbidge, E. M.; Burbidge, G. R.; Fowler, W. A.; Hoyle, F. (1957)."Synthesis of the Elements in Stars". Reviews of Modern Physics 29 (4): 547-650.

[2]. J. J. Griffin, Phys. Rev. Lett. 17(1966)478.

[3]. 3. A. A. Selman, " Neutron Induced Preequilibrium Nuclear Reactions Using the Exciton Model", Ph.D. Thesis, Uni. Baghdad, (2009).

[4]. C. K. Cline and M. Blann, Nucl. Phys. A172(1971)225.

[5]. E. Běták and P. E. Hodgson, "Particle-Hole State Density in Pre-Equilibrium Nuclear Reactions," University of Oxford, Report reference OUNP-98-02 (1998)

[6]. F. C. Williams, Jr., Nucl. Phys. A166(1971)231.

[7]. Arnould, M.; Goriely, S. (2003). "The p-Process of Stellar Nucleosynthesis: Astrophysics and Nuclear Physics Status". Physics Reports 384 (1-2): $1-84$ 